BULL. AUSTRAL. MATH. SOC.

VOL. $2(1970), 107-115$.

\title{
On commutativity in certain rings
}

\section{H. G. Moore}

I.N. Herstein has shown that an associative ring in which the nilpotent elements are "well-behaved", and such that every element satisfies a certain polynomial identity, is commutative. This result is generalized here. Specifically, it is shown that an alternative ring $R$ which satisfies the following three properties is commutative:

(i) for $x \in R$, there exists an integer $n(x)$ and a polynomial $p_{x}(t)$ with integer coefficients such that $x^{n+1} p(x)=x^{n}$;

(ii) for a fixed positive integer $m, a$ a nilpotent and $b$ an arbitrary element of $R, a-a^{m}$ commutes with $b-b^{m}$;

(iii) for the same $m, a$ and $b,(a b+b)^{m}=(b a+b)^{m}$ and $(a b)^{m}=d^{m} b^{m}$.

Examples are given to show that all three properties are essential, and it is shown that for associative rings certain modiried versions of these properties are individually enough to assure that the commutator ideal of the ring is nil.

\section{Introduction}

The beautiful theorem of Jacobson, which generalized the classical Wedderburn theorem on finite division rings, by proving that any

Received 24 October $196 \dot{9}$. 
assoclative ring in which $x^{n(x)}=x$ for all $x$, is a commutative ring [3], has itself become the spring board for several generalizations; all of these asserting that an appropriately conditioned ring is commutative or nearly so. In particular, Herstein has shown that an associative ring in which the nilpotent elements are "well-behaved" and such that every element satisfies a certain polynomial identity, is commutative [2]. In this paper Herstein's result is generalized. Additional results are obtained by modifying certain of the conditions of this generalization. We show that in these cases the ideal $C(R)$ generated by the commutators $x y-y x$ of elements of an associative ring $R$, is a nil ideal in $R$, so that such a ring $R$ is "almost commutative".

More specifically, we prove the following theorem, the case $m=1$ of which recovers Herstein's theorem:

THEOREM 1. Let $R$ be an altemative ring and let $N$ be the set of nilpotent elements of $R$. Let $m$ be an arbitrary but fixed positive integer. If, furthermore,

(i) for every $x$ in $R$ there exists an integer $n=n(x)$ and a polynomial $p(t)=p_{x}(t)$ with integer coefficients such that $x^{n+1} p(x)=x^{n}$,

(ii) $a \in N$ and $b \in R$ imply $a-a^{m}$ commutes with $b-b^{m}$,

(iii) $a \in N$ and $b \in R$ imply $(a b+b)^{m}=(b a+b)^{m}$ and $(a b)^{m}=d^{m} b^{m}$.

then $R$ is commutative.

By means of counter examples we shall show that the deletion of any one of hypotheses (i), (ii), or (iii) in Theorem 1 is enough to destroy commutativity in $R$ (even if $R$ is associative).

\section{Commutativity theorem}

In preparing for the proof of Theorem 1, we establish the following lemmas.

LEMMA 1. Let $R$ be an alternative ring, and let $m$ be a fixed 
positive integer. If, for any nilpotent element $a$ of $R$ and any $b \in R, a-a^{m}$ commutes with $b-b^{m}$, then the nilpotent elements of $R$ commute with each other.

Proof. Recall that by the Artin theorem any subring generated by any two elements of an alternative ring $R$ is associative. Denote the set of nilpotent elements of $R$ by $N$. Suppose $a \in N, b \in R$. Then $b-b^{m}$ commutes with each of $a-a^{m}, a^{m}-a^{m^{2}}, \ldots, a^{m^{k-1}}-a^{m^{k}}$, and hence $b-b^{m}$ commutes with the sum of these elements. Thus, $b-b^{m}$ commutes with $a-a^{m^{k}}$ for every positive integer $k$. Now, choose $k$ so large that $a^{m^{k}}=0$. Then $b-b^{m}$ commutes with $a$. We have thus shown that

$$
a\left(b-b^{m}\right)=\left(b-b^{m}\right) a \quad(a \in N, b \in R)
$$

Replacing $b$ by $b^{m}, b^{m^{2}}, \ldots, b^{m^{r-1}}$, and adding, we obtain

$$
a\left(b-b^{m^{r}}\right)=\left(b-b^{m^{r}}\right)_{a} \quad(a \in N, b \in R, r \geq 1) \text {. }
$$

The lemma now readily follows from (2) upon choosing $b \in N$ and $r$ so large that $b^{m^{r}}=0$.

LEMMA 2. Let $R$ be an alternative ring and let $N$ be the set of nilpotent elements of $R$. Let $m$ be a fixed positive integer. If,

(i) $a \in N$ and $b \in R$ imply $a-a^{m}$ commutes with $b-b^{m}$,

(ii) $a \in N$ and $b \in R$ imply $(a b+b)^{m}=(b a+b)^{m}$ and $(a b)^{m}=a^{m} b^{m}$, then every nilpotent element of $R$ is in the center of $R$.

Proof. Let $a \in N$ and let $b$ be an arbitrary element of $R$. Then, by (ii), $(a b)^{m}=a^{m} b^{m}$ and hence $(a b)^{m^{k}}=a^{m^{k}} b^{m^{k}}$ for all positive integers $k$. Therefore, $a \in N$ implies $a b \in N$. Since $(b a)^{r}=b(a b)^{r-1} a$, it further follows that $a \in b$ implies $b a \in N$. We have thus shown that 
Now, by Lemma 1 , it is easily seen that $a \in N, b \in N$ imply that $a b=b a ;$ and hence, $a-b \in N$.

Thus, by (3) and (4), we see that all products consisting of $i a^{\prime}$ s together with $j b^{\prime}$ s in eny order are equal provided that $i \geq 2$, $a \in N, b \in R$.

Hence, for any $a \in N, b \in R$, it follows that

$$
\begin{aligned}
& (a b+b)^{m}=b^{m}+a b^{m}+\sum_{i=1}^{m-1} b^{i} a b^{m-i}+\sum_{i=2}^{m}\left(\begin{array}{l}
m \\
i
\end{array}\right) a^{i} b^{m}, \\
& (b a+b)^{m}=b^{m}+b^{m} a+\sum_{i=1}^{m-1} b^{i} a b^{m-i}+\sum_{i=2}^{m}\left(m_{i}^{m}\right) a^{i} b^{m} .
\end{aligned}
$$

Therefore,

$$
(a b+b)^{m}-(b a+b)^{m}=a b^{m}-b^{m} a \quad(a \in N, b \in R) .
$$

Thus, by (1) and (5) and hypothesis (ii),

$$
a b-b a=0 \quad(a \in N, b \in R),
$$

and the lemma is proved.

In the proof of Lemma 2 we have also proved the following:

COROLLARY. Let $R, N, m$ be as in Lemma 2, and let (i) and (ii) hold. Then $N$ is an ideal in $R$. Furthermore, $N$ (as a ring) is conmutative.

Next, we state Herstein's Theorem [2] which plays an essential role in the proof of Theorem 1 .

THEOREM 2. Let $R$ be an associative ring such that for every element $x$ in $R$ there exists a positive integer $n=n(x)$ and $a$ polynomial $p(t)=p_{x}(t)$ with integer coefficients such that $x^{n+1} p(x)=x^{n}$. If, further, all the nilpotent elements of $R$ are in the center of $R$ then $R$ is comrutative.

We are now in a position to prove Theorem 1 .

Proof of Theorem 1. Let $a, b \in R$ and let $S$ be the subring of $R$ 
generated by $a$ and $b$. By Lemma 2, every nilpotent element of $R$ is in the center of $R$, hence, a fortiori this is true of $S$. Thus, by Theorem 2, $S$ is commutative so $a b=b a$, and Theorem $I$ follows.

Theorem 2 is the case $m=1$ of Theorem 1 , when $R$ is associative.

\section{Remarks and examples}

Consider the following examples which show that Theorem 1 need not hold if any one of the hypotheses (i), (ii), (iii) is deleted, even if $R$ is associative.

EXAMPLE 1. Let $R$ be the real quaternions, and let $m$ be any fixed positive integer. Here $R$ satisfies (ii) and (iii) but (i) fails to hold. Moreover, $R$ is not commutative. Thus, commutativity fails if hypothesis (i) of Theorem $I$ is deleted.

EXAMPLE 2. Let $R$ and $m$ be defined by

$$
R=\left\{\left[\begin{array}{lll}
a & b & c \\
0 & a & d \\
0 & 0 & a
\end{array}\right] ; a, b, c, d, \in \mathrm{GF}(3)\right\}, m=3 .
$$

It is readily verified that $R$ satisfies (i) and (iii) but not (ii). Moreover, $\dot{R}$ is not commutative. Thus, commutativity does not necessarily follow if hypothesis (ii) of Theorem 1 is deleted.

EXAMPLE 3. Let $R$ and $m$ be defined by

$$
R=\left\{\left[\begin{array}{ll}
0 & 0 \\
0 & 0
\end{array}\right],\left[\begin{array}{ll}
0 & 1 \\
0 & 1
\end{array}\right],\left[\begin{array}{ll}
1 & 0 \\
1 & 0
\end{array}\right],\left[\begin{array}{ll}
1 & 1 \\
1 & 1
\end{array}\right] ; 0,1 \in \mathrm{GF}(2)\right\}, m=2 .
$$

Here $R$ satisfies ( $i$ ) and (ii) but (iii) fails to hold. Since, moreover, $R$ is not commutative, hypothesis (iii) of Theorem 1 cannot be deleted.

It is worth observing that the ring $R$ of Example 3 satisfies:

$$
\left(x-x^{2}\right)\left(y-y^{2}\right)=0 \text { and }(x y)^{2}=x^{2} y^{2} \text { for all } x, y \text { in } R \text {. }
$$

This, in turn, shows that the first condition in hypothesis (iii) of Theorem 1 cannot be deleted even if both $(i i)$ and the second condition in (iii) were strengthened by assuming them to hold "globally" in $R$.

\section{A modified condition ( $i i i$ )}

The importance of condition (iii) of Theorem 1 was demonstrated by 
Example 3. In this section we show that a condition like the first part of (iii) will force the commutator ideal $C(R)$ of an associative ring $R$ to be nil, and when $R$ is semi-simple will force $R$ to be commutative.

Let $R$ be a ring which satisfies the following property:

Property P. $R$ is associative, and for every pair $a, b$ of elements of $R$ there exist positive integers $m(a, b)$ and $n(a, b)$ such that

$$
(a b+b)^{m(a, b)}=(b a+b)^{n(a, b)} .
$$

It is easy to see that no complete matrix ring $M_{k}(D)$ over a division ring $D$ (or over any associative ring, $D$, with unity) satisfies Property $P$ when $k>1$. As an example, let $a$ be the matrix unit $E_{1 k}$ while $b=\left(b_{i j}\right)$ with $b_{1 j}=1, j<k, b_{1 k}=0$ and $b_{i j}=0$ $i>1$. Then $(a b+b)^{n}=b$, and $(b a+b)$ is idempotent.

If $R$ has a unity, then $R$ is a generalized commutative ring as discussed by Belluce, Herstein and Jain in [1], since in this case Property $P$ becomes

$$
[(a+1) b]^{m}=[b(a+1)]^{n} \text {, }
$$

and if we set $c=a+1$, then as $a$ and $b$ run over $R$, so do $c$ and $b$. Therefore, from [1, Theorem 1] we have

LEMMA 3. If $R$ is a division ring which satisfies Property $P$, then $R$ is comutative.

It is an easy matter to use the structure theory now, since homomorphic images and subrings of rings which have Property $P$ also have this property, to obtain

THEOREM 3. If $R$ is a semi-simple ring with Property $\mathrm{P}$, then $R$ is commutative.

COROLLARY. Let $R$ be a ring with Property $\mathrm{P}, J(R)$ its Jacobson radical, and $C(R)$ its commutator ideal. Then $C(R) \subseteq J(R)$.

The final result is then proved by adapting the proof of Theorem 3 of [1]. We omit the details. 
THEOREM 4. Let $R$ be an associative ring such that for each pair $a, b$ of elements of $R$ there exist positive integers $m=m(a, b)$ and $n=n(a, b)$ such that $(a b+b)^{m}=(b a+b)^{n}$. Then the commutator ideal of $R$ is niz.

\section{Modification of condition (ii)}

A modification of condition ( $i i)$ of Theorem 1 similar to that of the previous section results in $C(R)$ being nilpotent provided that the associative ring $R$ also satisfies the descending chain condition for left ideals (d.c.c.). The result is contained in the next theorem, where, as before, $J$ is the Jacobson radical, $C$ the commutator ideal, and $N$ the set of all nilpotent elements of $R$.

THEOREM 5. Let $R$ be an associative ring such that

(i) $R$ satisfies the d.c.c.,

(ii) $R / J$ is finite, and

(iii) for each $a \in N$ and $b \in R$ there exist integers $n=n(a, b)>1$ and $m=m(a, b)>1$ such that $a-a^{n}$ commites with $b-b^{m}$.

Then $R / J$ is commutative; hence $C(R) \subseteq J$ and $C(R)$ is nilpotent.

Proof. Since $R$ satisfies the d.c.c., $J$ is nilpotent. Therefore, the ring $R / J$ also satisfies part (iii) of the hypothesis of this theorem. If $R / J$ is a division ring, then, being: finite, it is cormutative by Wedderburn's theorem. Since in any case, $R / J$ is semi-simple and finite, it is the complete direct sum of complete matrix rings $M_{k_{i}}\left(\Delta_{i}\right)$ over division rings $\Delta_{i}$. But for $k_{i}>1$, such matrix rings fail to satisfy (iii); yet they as direct summands must do so. To see this, take $a$ and $b$ to be the elements of $R / J$ whose $i$-th components are the matrices $E_{1 k}$ and $E_{k l}$, respectively, and for $j \neq i$ the components are 0 . Then $a^{2}=b^{2}=0$, and $a b \neq b a$. Therefore, $R / J$ must be a direct sum of fields, and we have $R / J$ commutative and $C(R) \subseteq J$. But $J$ is nilpotent, so a fortiori $C(R)$ is nilpotent as desired. 
The well-known result that a finite ring with no non-zero nilpotent elements is commutative is then, not only a corollary to Theorems $I$ and 2 , but to Theorem 5 as well.

A slightly different modification of condition (ii) of Theorem 1 which does not need d.c.c. is

THEOREM 6. Let $R$ be an associative ring with the property that for each pair of elements $a, b \in R$ there exist integers $m=m(a, b)>1$ and $n=n(a, b)>I$ such that

$$
\left(a^{m}-a\right)\left(b^{n}-b\right)=0 \text {. }
$$

Then the commutator ideal $C(R)$ of $R$ is nil and if $R$ is semi-simple $(J(R)=0)$, it is commutative.

Proof. We note first that (6) implies $\left(x^{m}-x\right)^{2}=0$ for all $x \in R$. Again we use the structure theory for the proof. If $R$ is a division ring $\left(x^{m}-x\right)^{2}=0$ implies $x^{m}-x=0$ and commutativity follows from Jacobson's Theorem. If we take $k>1$ the ring $M_{k}(D)$ fails to satisfy (6) for if $a=E_{1 k}$ and $b=E_{k l}$ then $a^{2}=b^{2}=0$, so $(-a)(-b)=a b=E_{11} \neq 0$.

Since (6) is hereditary, any primitive ring which satisfies it is a division ring; and, therefore, commutative. Thus, if $R$ is semi-simple $(J(R)=0)$ it is commutative.

Now $R / J$ is commutative, for arbitrary $R$ satisfying (6), so $C(R) \subseteq J$. We show that for any $a \in J, a^{2}=0$, which will complete the proof. Let $a \in J$. Then (for $a=b$ ) $a^{m-1} \in J$. Let $c$ be the right quasi-inverse for $a^{m-1}$. Since $\left(a^{m}-a\right)^{2}=0$

$$
a^{2}\left(a^{m-1}-1\right)^{2}(c-1)^{2}=0
$$

or

$$
a^{2}=0 \text {. }
$$

This computation can be carried out formally if $R$ fails to have a unity. We conclude with the following corollary to Theorem 6 . 
COROLLARY [added on 10 December 1969]. If $R$ is any associative ring satisfying (6), then $J(R)=N$, the set of nilpotent elements of $R$. Furthermore, $J^{2}=N^{2}=0$.

Proof. We have shown above that $J(R) \subseteq N$. If $a \in N$, then from (6) we have $\left(a^{m}-a\right)^{2}=0$. Let $\lambda$ be the least integer such that $a^{\lambda}=0$. Then $\lambda \leq 2$, since otherwise we multiply $a^{2 m}-2 a^{m+1}+a^{2}=0$ by $a^{\lambda-3}$ obtaining

$$
a^{2 m+\lambda-3}-2 a^{m+1+\lambda-3}+a^{\lambda-1}=0 .
$$

(If $\lambda=3$ we have (7) immediately from (6).) But (7) becomes $a^{\lambda-1}=0$ contradicting the minimality of $\lambda$. Thus $a^{2}=0$ for all $a \in N$. Now if $a \in N$ and $b$ is arbitrary, $(a b-b a)^{2}=0$; whence,

$$
(a b)^{2}+(b a)^{2}-a b^{2} a=0 .
$$

Multiplying on the left by $a$ gives

$$
a(b a)^{2}=(a b)^{2} a=0 .
$$

Whence, $(a b)^{3}=0$; so $a b \in N$ and $a b$ is right quasi-regular for each $b \in R$. Thus, $a \in J(R)$ and we have equality as desired. The last statement of the corollary now follows immediately from (6).

\section{References}

[1] L.P. Belluce, I.N. Herstein and S.K. Jain, "Generalized commutative rings", Nagoya Math. J. 27 (1966), 1-5.

[2] I.N. Herstein, "A note on rings with central nilpotent elements", Proc. Amer. Math. Soc. 5 (1954), 620.

[3] Nathan Jacobson, Structure of rings (Colloquium Publ. 37, Amer. Math. Soc., Providence, revised ed., 1964).

[4] Richard D. Schafer, An introduction to nonassociative algebras (Academic Press, New York and London, 1966).

Brigham Young University, Provo, Utah, USA. 